Supporting Information

\title{
Probing The Active Site Of An Azurin Mutant Hot- Wired To Gold Electrodes
}

Authors:

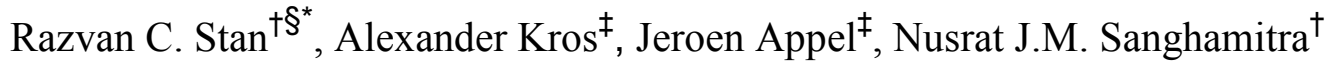

\begin{abstract}
Affiliations:
${ }^{\dagger}$ Leiden Institute of Physics-Huygens Laboratories, Leiden University, P.O. Box 9504, 2300 RA, Leiden, The Netherlands

‡ Leiden Institute of Chemistry-Gorlaeus Laboratories, Leiden University, P.O. Box 9502, 2300 RA, Leiden, The Netherlands

$\S$ Present address: Institute for Biomedical Sciences, University of São Paulo, CEP 05508-900, São Paulo, Brazil

* Please address all correspondence to Razvan Stan, strazvan@usp.br

The accompanying report includes 1. TM-AFM and C-AFM images of single native azurins and azurin H117G-Cu-Linker 1 complexes adsorbed on gold surfaces, 2. ellipsometric measurements and data fitting of two azurin monolayers, 3. Scanning Electron Microscopy (SEM) imaging of AFM probes post acquisition of $\mathrm{I}(\mathrm{V})$ data.
\end{abstract}




\section{TM-AFM and C-AFM imaging of single wt azurin and azurin complexes.}
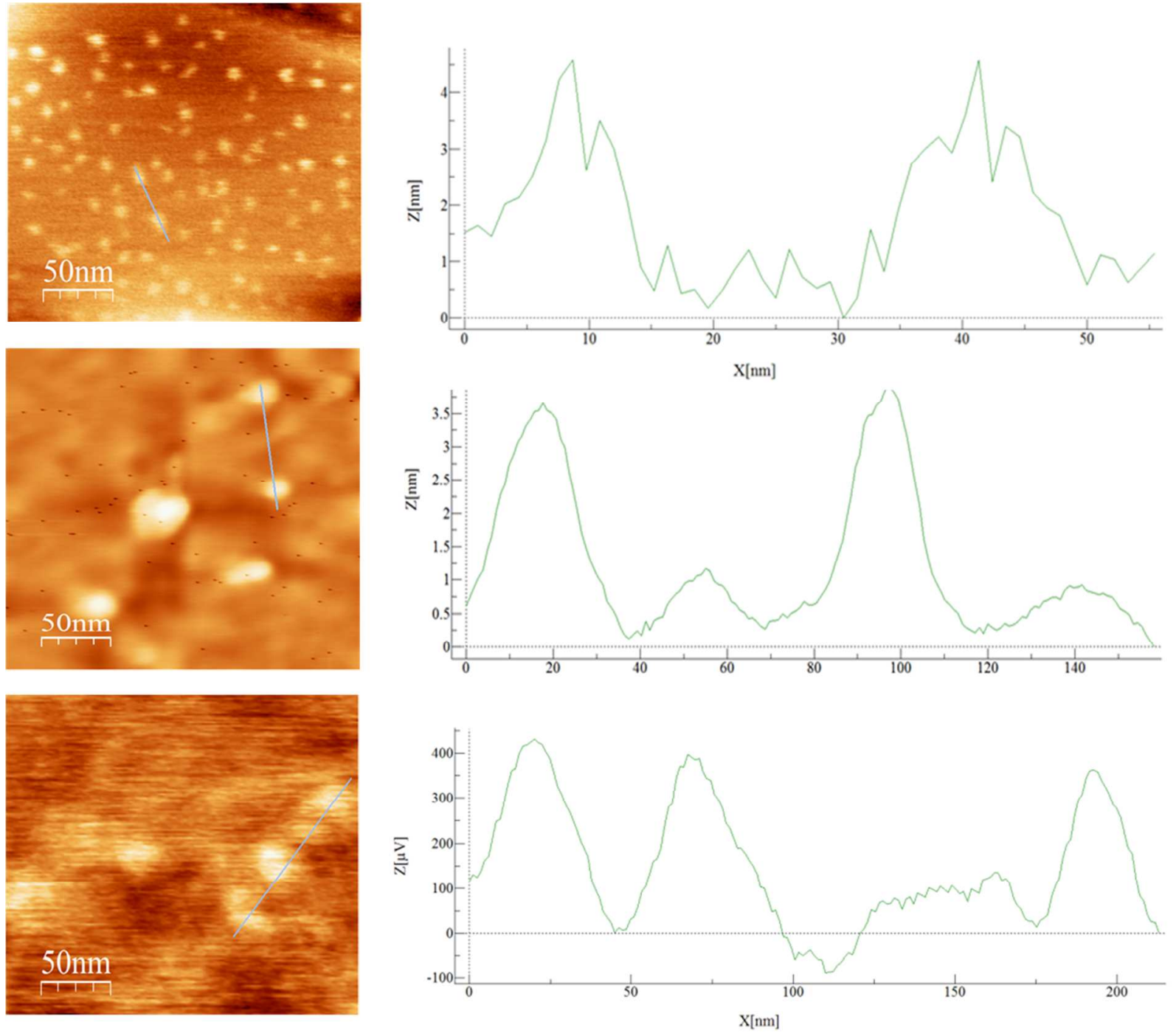

Figure S1. a. TM-AFM image of submonolayers of azurin $\mathrm{H} 117 \mathrm{G}-\mathrm{Cu}(\mathrm{II})$ reconstituted with Linker 1 and chemisorbed to gold surfaces. b. TM-AFM image of Au-adsorbed submonolayers of native azurin. Cross-sections indicate the vertical dimension of individual wild type/reconstituted azurin complexes. c. C-AFM image of azurin H117G-Cu(II) reconstituted with Linker 1. For all images, surface preparation consisted of short incubation times (30") of the proteins on the gold electrodes, followed by rinsing with MES buffer, Mili-Q water and drying with Ar. Images prepared using WSxM 4.0 (WSxM Solutions, Spain). 


\section{Ellipsometry of adsorbed azurin films.}

A M2000V Variable Angle Spectroscopic Ellipsometer (Woollam) was used for characterization with the incident light at a fixed angle of $65^{\circ}$. The illuminating arm consisted of a 50W quartz tungsten halogen lamp, while the detection arm permits spectroscopic measurements within the 370-1000 nm range. Analysis of the data was performed with the WVase software package (Woollam, Lincoln, USA).
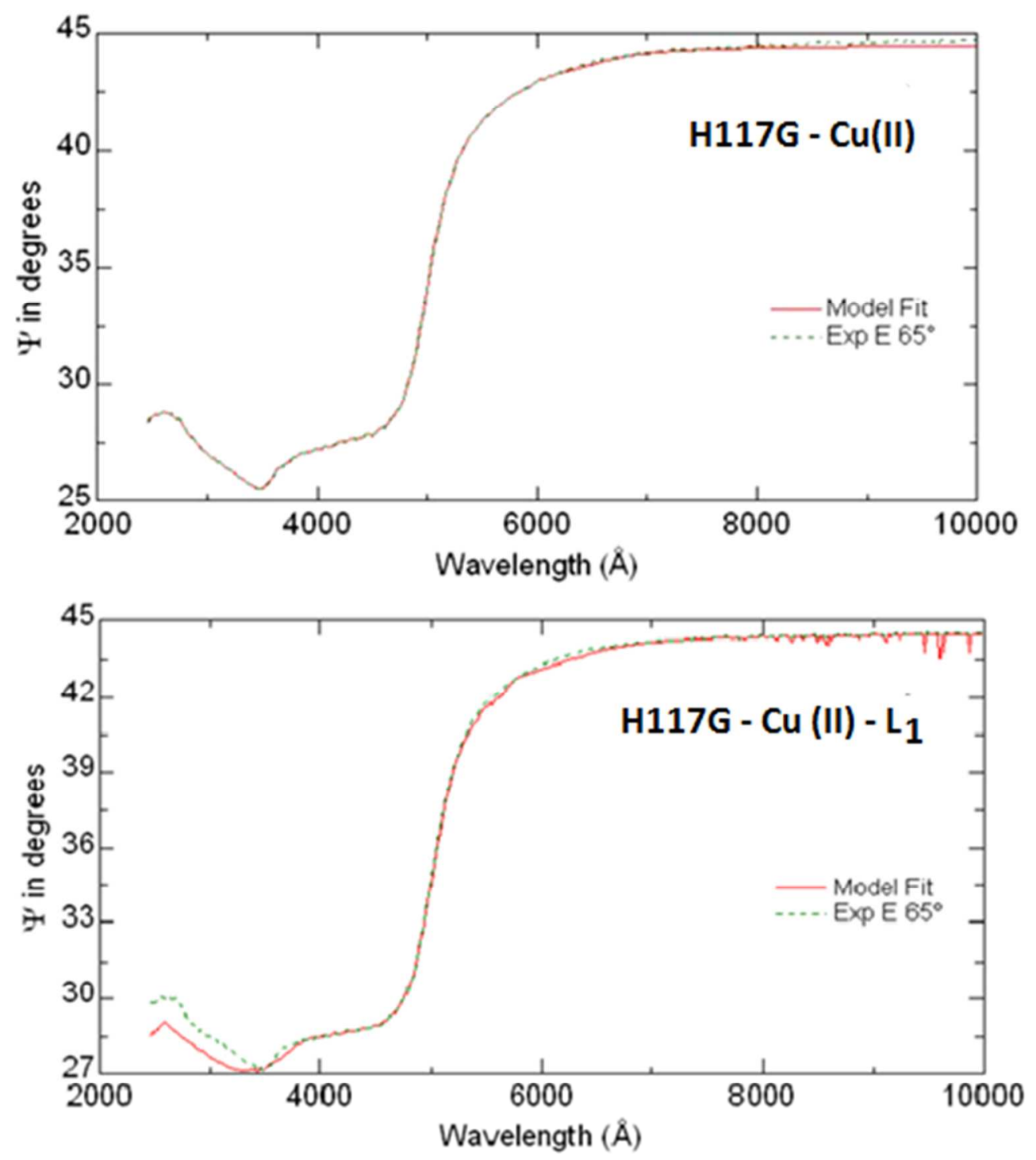

Figure S2. Examples of ellipsometrical measurements (dotted line, MSE below 1), and data fitting of the holoazurin assembled on gold (above) and of azurin reconstituted with Linker 1 (below). 


\section{SEM measurements of C-AFM probes.}

In order to ascertain the use of undamaged, uncontaminated and conductive cantilevers for our $\mathrm{I}(\mathrm{V})$ measurements, we have imaged the C-AFM probes after sampling the conductivity of the protein films. Four examples of such probes are presented in figure S2.
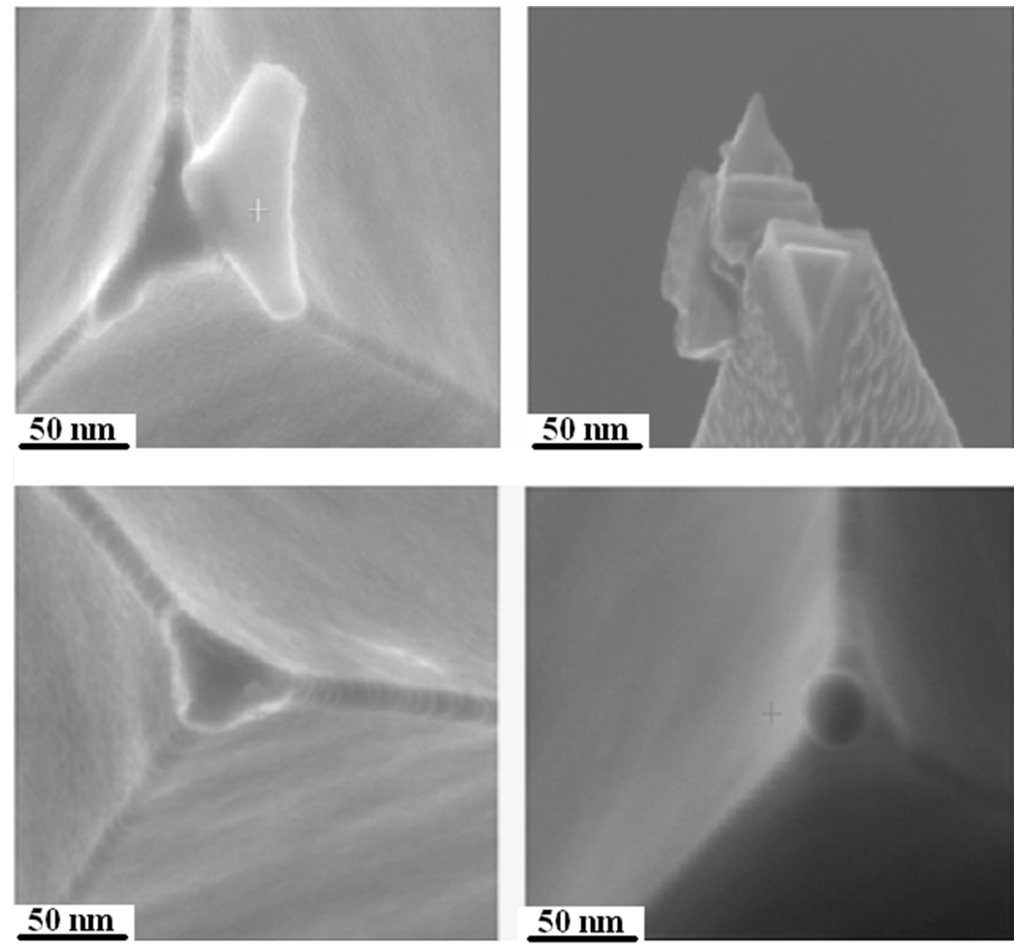

Figure S3. Top-view SEM micrographs of C-AFM cantilevers used for force-calibration and for performing current-voltage spectroscopy. Top row: images of damaged, non-conductive tips after scanning the azurinfunctionalized gold surfaces, with a lifetime of usually below 10'. Bottom row: conductive C-AFM tips after usage for I(V) spectroscopy performed at moderate forces $(5-10 \mathrm{nN})$.

It is evident that scanning functionalized surfaces has adverse effects on the integrity of the cantilever's conductive coating, which results in unstable imaging and unreliable I(V) spectra; as such, no data obtained with such cantilevers were used for analysis (fig. S3, top-row). In contrast, intact conductive cantilevers after having only been used for brief imaging followed by force-curve measurements and $\mathrm{I}(\mathrm{V})$ spectroscopy are reusable (bottom-row in figure $\mathrm{S} 3$ ). 